\title{
A Seed Mucilage-Degrading Fungus From the Rhizosphere Strengthens the Plant-Soil-Microbe Continuum and Potentially Regulates Root Nutrients of a Cold Desert Shrub
}

\author{
Dandan Hu, ${ }^{1,4}$ Jerry M. Baskin, ${ }^{2}$ Carol C. Baskin, ${ }^{2,3}$ Rong Liu, ${ }^{1,5}$ Xuejun Yang, ${ }^{1,+}$ and Zhenying Huang ${ }^{1, \dagger}$ \\ ${ }^{1}$ State Key Laboratory of Vegetation and Environmental Change, Institute of Botany, Chinese Academy of Sciences, Beijing \\ 100093, China \\ ${ }^{2}$ Department of Biology, University of Kentucky, Lexington, KY 40506, U.S.A. \\ ${ }^{3}$ Department of Plant and Soil Sciences, University of Kentucky, Lexington, KY 40546, U.S.A. \\ ${ }^{4}$ Key Laboratory for Humid Subtropical Eco-Geographical Processes of the Ministry of Education, School of Geographical \\ Sciences, Fujian Normal University, Fuzhou 350007, China \\ ${ }^{5}$ University of Chinese Academy of Sciences, Beijing 100039, China
}

Accepted 15 February 2021.

Seed mucilage plays important roles in the adaptation of desert plants to the stressful environment. Artemisia sphaerocephala is an important pioneer plant in the Central Asian cold desert, and it produces a large quantity of seed mucilage. Seed mucilage of $A$. sphaerocephala can be degraded by soil microbes, but it is unknown which microorganisms can degrade mucilage or how the mucilage-degrading microorganisms affect rhizosphere microbial communities or root nutrients. Here, mucilage-degrading microorganisms were isolated from the rhizosphere of $A$. sphaerocephala, were screened by incubation with mucilage stained with Congo red, and were identified by sequencing and phylogenetic analyses. Fungal-bacterial networks based on high-throughput sequencing of rhizosphere microbes were constructed to explore the seasonal dynamic of interactions between a mucilage-degrading microorganism and its closely related microorganisms. The structural equation model was used to analyze effects of the mucilage-degrading microorganism, rhizosphere fungal-bacterial communities, and soil physicochemical properties on root $\mathrm{C}$ and $\mathrm{N}$. The fungus Phanerochaete chrysosporium was identified as a mucilagedegrading microorganism. Relative abundance of the mucilagedegrading fungus (MDF) was highest in May. Subnetworks

Sequence data is available in the National Center for Biotechnology Information Sequence Read Archive database under accession number PRJNA602135.

${ }^{\dagger}$ Corresponding authors: X. Yang; xjyang_jx@ibcas.ac.cn and Z. Huang; zhenying@ibcas.ac.cn

Funding: This work was supported by the National Natural Science Foundation of China (grant numbers 31861143024, 31870711, 31770514 and 32071524) and the Key Basic Research and Development Plan of China (2016YFC0500805-02).

*The $\boldsymbol{e}$-Xtra logo stands for "electronic extra" and indicates there is supplementary material published online.

The author(s) declare no conflict of interest.

(c) (1) $\circledast \Theta$ Copyright $\odot 2021$ The Author(s). This is an open access article distributed under the CC BY-NC-ND 4.0 International license. showed that the abundance of fungi and bacteria closely related to the MDF also were highest in May. Interactions between the MDF and related fungi and bacteria were positive, which might enhance mucilage degradation. In addition, the MDF might regulate root $\mathrm{C}$ and $\mathrm{N}$ by affecting rhizosphere microbial community structure. Our results suggest that $\mathrm{MDF}$ from the rhizosphere strengthens the plant-soil-microbe continuum, thereby potentially regulating microbial interactions and root nutrients of $A$. sphaerocephala.

Keywords: mucilage-degrading fungus, rhizosphere bacteria, rhizosphere fungi, root $\mathrm{C}$, root $\mathrm{N}$, seed mucilage

Seed traits related to dormancy and germination strategies are significant contributors to adaptation to environmental variation and long-term species demographics under natural conditions, as reported in a guild of coexisting species in the desert (Huang et al. 2016; Liu et al. 2020). Seed mucilage is such a trait that plays important roles in helping desert plants adapt to the variable environment (Gutterman et al. 1973). Seed mucilage is a mucilaginous layer on the external surface of seed coats that is synthesized during seed development. After imbibing water, seed mucilage forms a pectinaceous layer with remarkable water-absorption and water-retaining capacities (Francoz et al. 2015; Grubert 1974; Yang et al. 2012a). Seed mucilage promotes seedling emergence and decreases seedling mortality by increasing water and oxytgen absorption and maintaining a moist environment, thereby promoting successful seedling establishment in sandy deserts (Gutterman et al. 1973; Yang et al. 2012a and b).

As a pectinaceous polysaccharide, seed mucilage can be degraded to small molecular weight monosaccharides by soil microbes, and the degradation products, such as glucose, mannose and arabinose, are directly utilized by seedlings to improve their growth in barren deserts (Yang et al. 2012b). In turn, the soil microbial community can utilize these monosaccharides as substrates, thereby increasing microbial biomass and exerting a beneficial effect on the soil microbial community (Yang et al. 2012b). In addition, the mucilage biodegradation products strengthen fungal-bacterial interactions and reduce resource competition between fungi and bacteria by providing a carbon source (Hu et al. 2019). 
Soil microbes are the major decomposers of organic matter and thus play an important role in biogeochemical cycles in ecosystems (López-Mondéjar et al. 2018). They can degrade lignin, cellulose, hemicellulose, pectin, and protein by secreting a large number of enzymes (Yadav and Malanson 2007). In semiarid areas, strains of Streptomyces and Saccharothrix produce large amounts of extracellular enzymes, such as cellulases, glucosidases, xylanases, and arabinosidases, that degrade cellulose and hemicellulose in the cell walls of grasses (Mohammadipanah and Wink 2016; Yeager et al. 2017). In addition, some Actinomycetes can degrade complex polysaccharides by secreting lignocellulosic hydrolase. For example, strains belonging to families Streptomycetaceae, Pseudonocardiaceae, Micromonosporaceae, and Promicromonosporaceae have a strong ability to degrade cellulose, xylan, chitin, and fructan (McCarthy 1987; Yeager et al. 2017). In the process of microbial degradation, degradation products provide a carbon source for the growth and activities of microbes, thereby affecting the microbial community structure (Banerjee et al. 2016; de Graaff et al. 2010). At the same time, nutrients released during microbial degradation can be used by plants for growth, and the nutrients can participate in biogeochemical cycles and affect carbon storage in the ecosystem (Schneider et al. 2012).

Microbes do not exist in isolation but form complex ecological associations (de Boer 2017; Deveau et al. 2018; Faust and Raes 2012). Complex positive (commensalism, mutualism) and negative (amensalism, parasitism, predation, competition) interactions occur in microbial communities (de Menezes et al. 2017; Deveau et al. 2018). de Boer et al. (2005) showed that fungi are the main decomposers for recalcitrant organic matter, while bacteria play an important role in the degradation of simple substrates. Fungi but not bacteria secrete polymerdegrading enzymes that decompose leaf litter (Schneider et al. 2012). Saprophytic bacteria compete with fungi for substrates and inhibit fungal degradation (de Menezes et al. 2017). However, bacteria also interact with fungi, thereby facilitating litter decomposition (de Boer et al. 2005). Therefore, the interactions between bacteria and fungi during degradation are complicated. To identify such complex interactions, correlation network analysis methods have been widely used to determine potential fungal-bacterial interactions and co-occurrence patterns in microbial communities (Banerjee et al. 2016; Creamer et al. 2016; de Menezes et al. 2017; Zhou et al. 2011); however, they are subject to some biases in providing reliable information on direct biotic interactions in real-world ecosystems (Carr et al. 2019).

The rhizosphere is a biologically active zone of the soil around roots, and rhizosphere microbes are more closely related to plants than microbes in the bulk soil (Berendsen et al. 2012, Mendes et al. 2013). Rhizosphere microbes play key roles in nutrient acquisition and assimilation that promote plant growth (Hayat et al. 2010). Plants modulate the rhizosphere microbial communities by selectively recruiting microorganisms beneficial to plant growth and health (Cook et al. 1995). The rhizosphere may harbor microorganisms regulating seed germination or seedling growth, including those that degrade seed mucilage. Our previous reports have shown that seed mucilage of A. sphaerocephala can be degraded by microbes in bulk soil (Yang et al. 2012b), but it is still unclear which soil microorganisms can degrade mucilage. Thus, we hypothesized that mucilage-degrading microorganisms exist in the rhizosphere and that they interact with other rhizosphere microbial species to affect mucilage degradation. To explore the seasonal dynamic of interactions between a mucilage-degrading microorganism and its closely related microorganisms, fungalbacterial networks based on the high-throughput sequencing of rhizosphere microbes in different seasons were constructed.

Many rhizosphere microbes are beneficial for plant growth by promoting nutrient absorption of plants (Hayat et al. 2010; Mendes et al. 2013). Members of the rhizosphere microbiota, such as nitrogen-fixing bacteria and mycorrhizal fungi, can rapidly convert and absorb various forms of $\mathrm{N}$ from the soil and subsequently relocate available $\mathrm{N}$ to roots (Mendes et al. 2013). Therefore, we hypothesized that seed mucilage-degrading microorganisms play an important role in regulating root nutrients of $A$. sphaerocephala by directly utilizing degradation products or indirectly affecting rhizosphere microbial community structure. To test this hypothesis, we used the structural equation model to analyze the effects of the mucilage-degrading microorganism, rhizosphere fungal-bacterial communities, and soil physicochemical properties on root $\mathrm{C}$ and $\mathrm{N}$.

\section{RESULTS}

Isolation of mucilage-degrading microorganisms.

During incubation, one dish of red mucilage began to fade after 2 weeks and had completely faded after 4 weeks (Fig. 1), indicating degradation of mucilage and the presence of mucilage-degrading fungi (MDF).

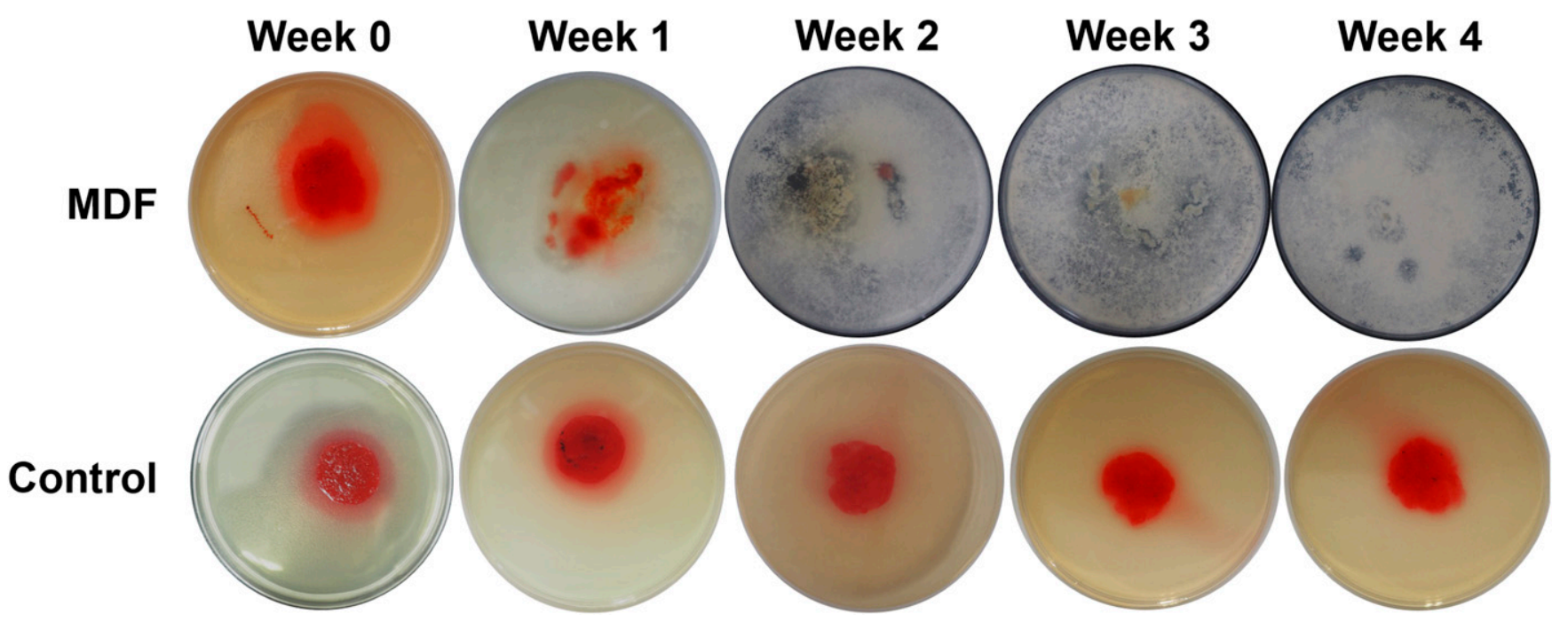

Fig. 1. Degradation of seed mucilage of Artemisia sphaerocephala by the mucilage-degrading fungus (MDF). 


\section{Molecular identification of the MDF.}

The intergenic transcribed spacer (ITS) sequence of the MDF was $695 \mathrm{bp}$, and it had been deposited in GenBank with the accession number MW003708 (Fig. 2A). The phylogenetic tree identified the MDF as Phanerochaete chrysosporium (strain code ATCC 24725, division Basidiomycota) (Fig. 2B). The relative abundance of the MDF was significantly higher in May than in July, September, and November $(P<0.05)$ (Fig. 2C).

\section{Diversity of rhizosphere fungal and bacterial communities among different seasons.}

For alpha diversity, operational taxonomic unit (OTU) richness and the Shannon index of bacterial communities differed significantly among seasons $(P<0.05)$. OTU richness of bacterial communities was the highest in May and November (Supplementary Fig. S3a) and was lowest in September, while the Shannon index of bacterial communities was lowest in July (Supplementary Fig. S3c). OTU richness of fungal communities were lowest in July (Supplementary Fig. S3b), whereas Shannon index of fungal communities were similar in different months (Supplementary Fig. S3d). For beta diversity, principal coordinate analysis (PCoA) revealed that the structure of both fungal and bacterial communities differed among months (Supplementary Fig. S3e and f). Permutational multivariate analysis of variance (PERMANOVA) confirmed that bacterial communities differed significantly among the four months, except between May and November $(P<0.05)$ (Supplementary Table S1). In addition, fungal communities differed significantly among the four months, except between September and November $(P<0.05$; Supplementary Table S1).

\section{MDF in the rhizosphere fungal-bacterial network.}

Topological properties of fungal-bacterial networks showed that the total number of links was highest in May (Supplementary Table S2). Subnetworks closely related to the MDF in the fungal-bacterial network showed that the number and type of fungi and bacteria closely related to MDF differed among seasons (Fig. 3; Supplementary Table S3). Microbial species closely related to MDF were highest and included 11 species of bacteria (two species from phylum Actinobacteria, two from

\section{A}

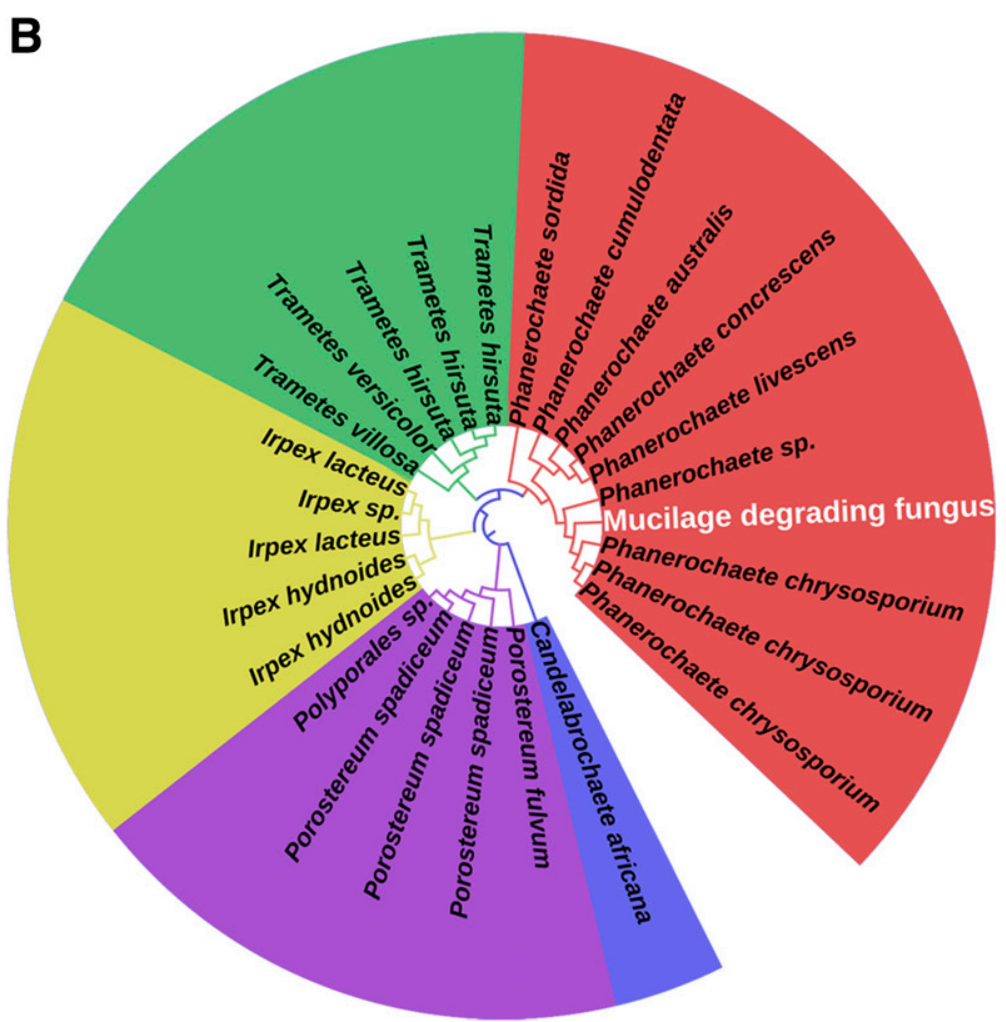

C

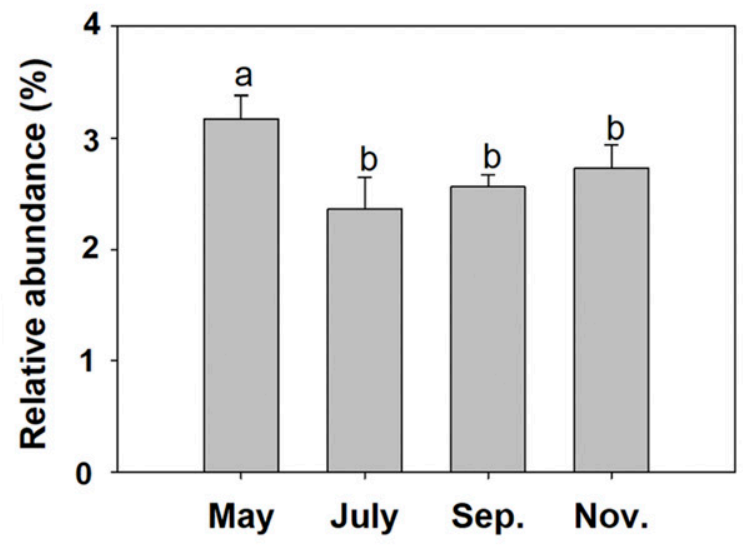

Fig. 2. A, Intergenic transcribed spacer (ITS) sequence, B, neighbor-joining tree based on phylogenetic analysis of ITS sequence data, and C, the relative abundance in different months of the seed mucilage-degrading fungus (MDF) from the rhizosphere of Artemisia sphaerocephala. Different lowercase letters indicate significant difference between months $(P<0.05)$. 
Firmicutes, six from Proteobacteria, and one from Verrucomicrobia) and one species of fungi (phylum Mucoromycota) in samples collected in May. All relationships between the MDF and these 11 closely related fungi and bacteria were positive in the fungal-bacterial subnetwork of May (Fig. 3E; Supplementary Table S3). One bacterium species of phylum Verrucomicrobia (B596) and two fungal species of phylum Basidiomycota (F173, F232) were closely positively related to the MDF and the other fungal species of Basidiomycota (F183) was closely negatively related to the MDF in samples collected in July. Only one species of phylum Ascomycota (F22) was closely negatively related to the MDF in samples collected in September (Fig. 3G; Supplementary Table S3). There were two species of phylum Ascomycota (F107, F131) closely positively associated with the MDF, and the other two species of Ascomycota (F93, F74) were closely negatively associated with the MDF in samples collected in November (Fig. 3H; Supplementary Table S3).

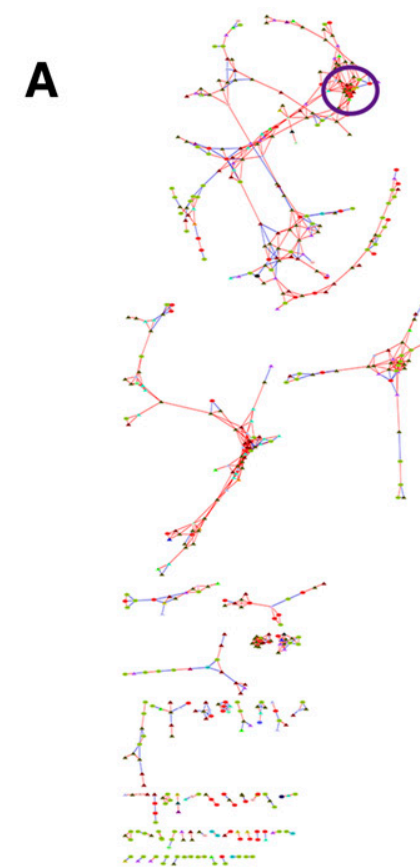

E

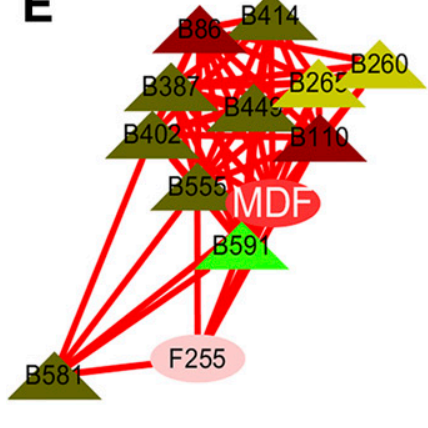

Fungi

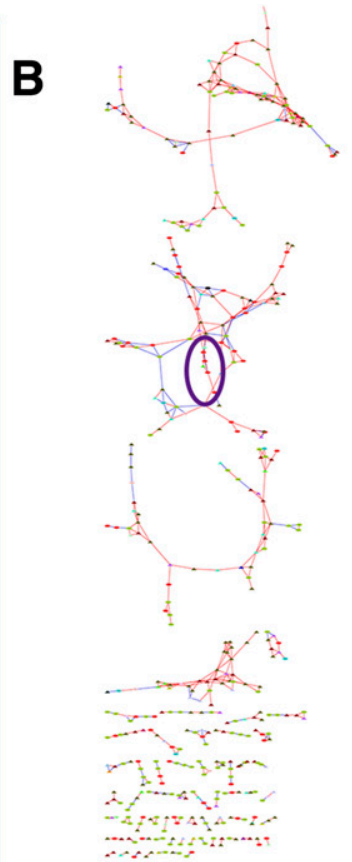

$\mathbf{F}$

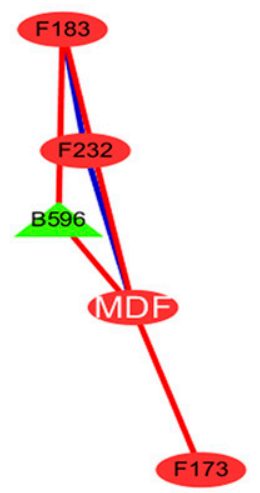

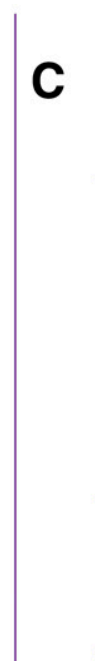

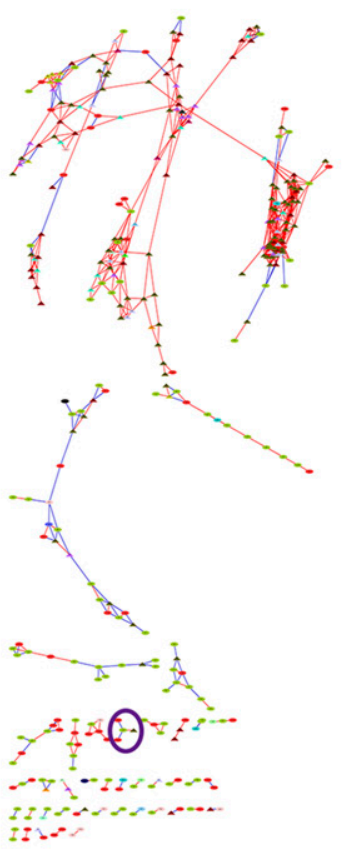

G

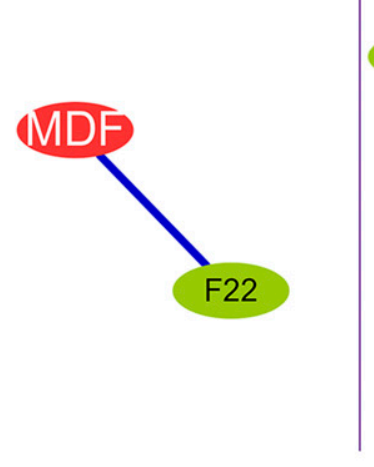

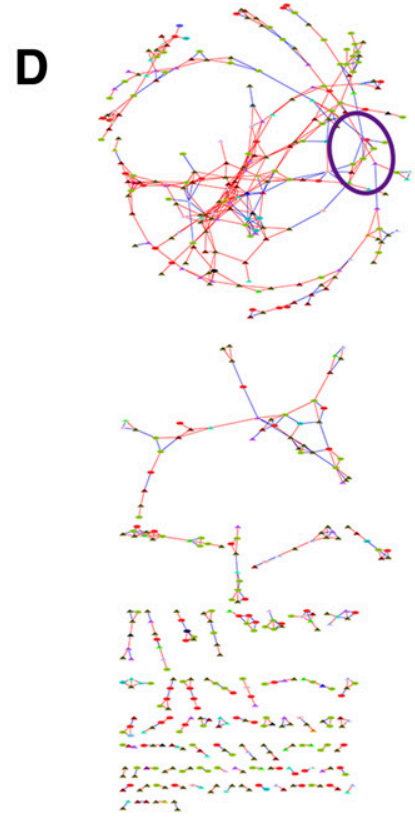

H

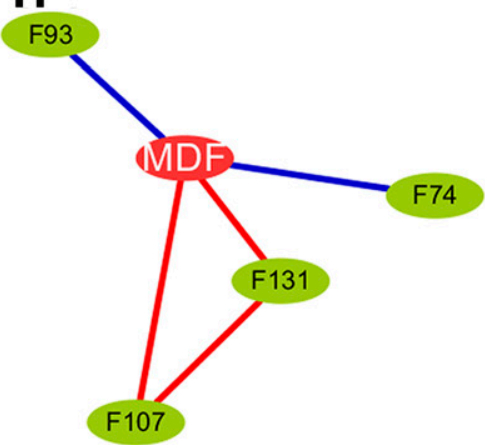

Ascomycota

Basidiomycota $\square$ Cercozoa

Chytridiomycota

Mortierellomycota

Mucoromycota

Rozellomycota

\section{Bacteria}

Acidobacteria

Actinobacteria

Armatimonadetes

Bacteroidetes

Chlorobi

Chloroflexi

Cyanobacteria

Deinococcus-Thermus Firmicutes

Gemmatimonadetes

Latescibacteria

Nitrospirae

Planctomycetes

Proteobacteria

Saccharibacteria

Fig. 3. Rhizosphere fungal-bacterial co-occurrence network and subnetwork of the connected hubs of the mucilage-degrading fungus (MDF) in A and E, May, $\mathbf{B}$ and $\mathbf{F}$, July, $\mathbf{C}$ and $\mathbf{G}$, September, and $\mathbf{D}$ and $\mathbf{H}$, November in the natural habitat of Artemisia sphaerocephala. Node shapes represent bacteria (triangle) and fungi (circle). Node colors indicate major bacterial and fungal phyla. Red lines represent positive co-occurrence relationships and blue lines negative relationships. 
Interactive effect of the MDF and soil fungi and bacteria on root $\mathbf{C}$ and $\mathbf{N}$.

Root carbon content of $A$. sphaerocephala was highest in May and lowest in July. The highest root nitrogen content was in May, and it decreased in July and September. Glucosidase, chitinase, and phosphatase activity were lowest in July (Supplementary Table S4). Structural equation models (SEM) explained $37 \%$ of the variation in root carbon content and $40 \%$ of root nitrogen content. Changes in the bacterial community had a direct positive effect on root carbon content (Fig. 4A). The fungal community had a direct negative effect on root nitrogen content, and MDF indirectly affected root nitrogen content by affecting the fungal community (Fig. 4A). SEM showed that the MDF and bacteria had the greatest influence on plant root carbon content (Fig. 4B). The MDF and fungi had the greatest influence on plant root nitrogen content (Fig. 4B).

\section{DISCUSSION}

The most important contribution of our research was the isolation and identification of the MDF Phanerochaete chrysosporium from the rhizosphere of A. sphaerocephala and the demonstration of its positive interactions with other closely related bacteria and fungi that might accelerate mucilage degradation. Thus, our hypothesis was supported. In addition, we
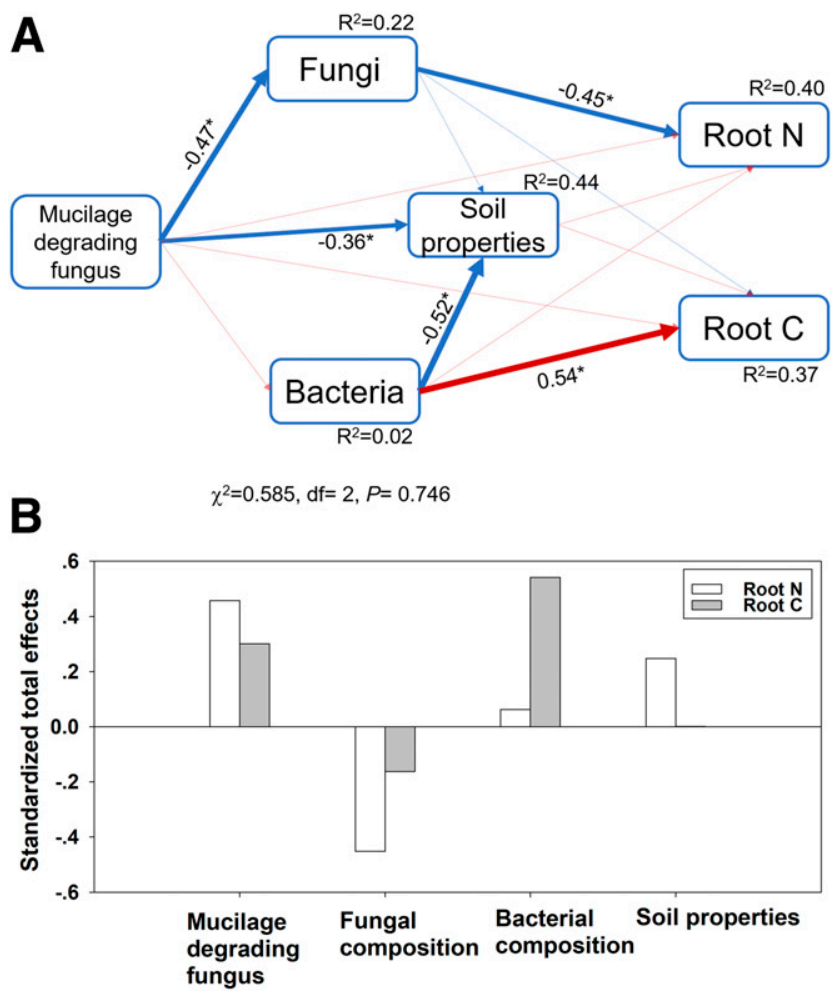

Fig. 4. Structural equation models (SEM) showing A, the direct and indirect effects of the seed mucilage-degrading fungus, rhizosphere fungal community, rhizosphere bacterial community, and soil chemo-physical properties on root $\mathrm{C}$ and $\mathrm{N}$ content of Artemisia sphaerocephala. B, Total standardized effects (direct plus indirect) of the mucilage-degrading fungus, fungal community composition, bacterial community composition and soil chemo-physical properties on root $\mathrm{C}$ and $\mathrm{N}$ contents derived from the SEM. Red and blue arrows indicate significantly positive and negative relationships, respectively, and dashed arrows nonsignificant relationships. Numbers adjacent to arrows are path coefficients, and width of the arrows is proportional to the strength of path coefficients, reflecting the effect size of the relationship. The proportion of variance explained $\left(R^{2}\right)$ appears alongside each response variable in the model. An asterisk (*) indicates a significance level of $P<0.05$. showed that the MDF increased the abundance of plant growth-promoting rhizobacteria (e.g., phylum Actinobacteria). We speculate that the MDF regulated root nutrients of A. sphaerocephala by affecting rhizosphere fungal and bacterial community structure.

\section{MDF in rhizosphere.}

Decomposer microorganisms are ubiquitous in soil (Rytioja et al. 2014). The seed mucilage of A. sphaerocephala consists of polysaccharides that can be decomposed by soil microbes (Yang et al. 2012b), but heretofore, it was unknown which microorganism or microorganisms can degrade mucilage. In this study, we screened a fungus from the rhizosphere that can degrade seed mucilage of A. sphaerocephala (Fig. 1). Further, we identified the MDF as Phanerochaete chrysosporium (phylum Basidiomycota) (Fig. 2B). Basidiomycota are known to degrade plant cell walls, and $P$. chrysosporium has a strong ability to degrade lignin (Baldrian and Valásková 2008).

P. chrysosporium secretes a variety of extracellular glycoside hydrolases that degrade structural polysaccharides such as cellulose and hemicellulose (Aro et al. 2005; Ray et al. 2012). In addition, $P$. chrysosporium secretes a variety of extracellular carbohydrate-hydrolyzing enzymes that degrade plant cell walls (Hori et al. 2011). The genome of $P$. chrysosporium contains genes encoding oxidase, peroxidase, and hydrolase (Martinez et al. 2004; Vanden Wymelenberg et al. 2009). In addition to degrading lignin, $P$. chrysosporium can secrete a large number of polysaccharide-degrading enzymes, which can degrade cellulose, hemicellulose, starch, glycogen, glycans, chitin, and dextran (Kameshwar and Qin 2017; Martinez et al. 2004).

\section{Relationships between the MDF and} other microorganisms in the rhizosphere.

The relative abundance of the MDF exhibited seasonal dynamics, and it was highest in May (Fig. 2C). Seeds of A. sphaerocephala are dispersed soon after maturity in November, after which seed mucilage would not be degraded due to low temperature and low precipitation in winter. In spring, seed mucilage can be degraded at suitable temperature and moisture conditions for microbial activities. The MDF $P$. chrysosporium reaches its highest relative abundance in May and can degrade seed mucilage quickly, thus supplying degradation products to seedlings of $A$. sphaerocephala for growth, as previously reported by Yang et al. (2012b).

By interacting with each other, fungi and bacteria accelerate the degradation of organic matter (de Boer et al. 2005; Rudnick et al. 2015). Saprophytic fungi provide a source of energy for the rhizosphere bacterial community, and bacteria obtain nutrients and energy from fungi by infecting fungal hyphae during lignin degradation (de Menezes et al. 2017; Hoppe et al. 2014). Nitrogen-fixing bacteria support lignocellulose fungal decomposers such as Clitocybe spp. and Mycena spp. by providing an available nitrogen source during leaf litter decomposition (Purahong et al. 2016). Phosphate solubilizing bacteria (genera Burkholderia, Flavobacterium, Pseudomonas, and Rhizobium) also provide $\mathrm{P}$ to fungal decomposers (Clitocybe spp. and Mycena spp.) in the early stages of litter decomposition (Rodríguez and Fraga 1999). By extracting the fungal-bacterial subnetworks, we found that species of fungi and bacteria closely related to the MDF varied among months (Fig. 3). Differences in the interactions of the microbial network over time have been reported in several studies. For example, network connections of an uncultured sulfate-reducing bacterium differed during the succession in microbial communities in response to environmental changes (Deng et al. 2016). Plant development stage (Shi et al. 2016) and seasonal dynamics of 
bulk soil biota (Oberholster et al. 2018) result in changes in the rhizosphere microbial interaction network over time. Furthermore, soil physiochemical properties such as soil temperature, moisture, and nutrients ( $\mathrm{N}$ and $\mathrm{P}$ ) were the key factors driving the seasonal dynamics of the rhizosphere bacterial communities in a Pinus tabulaeformis forest in the Qinling Mountains (Wang et al. 2018). Therefore, the differences in the interaction of the network with the MDF strain over time might be affected not only by the plant growth stage but also by the bulk soil biota and soil properties. The MDF was associated with the highest number of closely related fungi and bacteria in May (Supplementary Table S3), indicating that the closely related fungi and bacteria may be indirectly involved in seed mucilage degradation by interacting with MDF. Further, all relationships between the MDF and closely related fungi and bacteria were positive in the fungal-bacterial subnetwork (Fig. 3E), thus the degradation of seed mucilage might be enhanced via the positive interactions of various bacteria and fungi.

Fungi and bacteria in the subnetworks closely related to the MDF may play a role in bridging transmission of information. The key nodes and links embedded in different bacterial or functional groups of the network can link other functional bacteria in the networks. When subjected to stressful environments, the relevant functional bacteria spread rapidly through the key nodes and links and improve their adaptability to the environment (Halary et al. 2010; Schlüter et al. 2003). The MDF directly interacts with the plant growth-promoting bacteria, actinomycetes, and rhizobia (Supplementary Table S3). Actinomycetes protect plants from pathogens by producing antibiotics that inhibit pathogens (Hamedi and Mohammadipanah 2015). Rhizobium spp. convert molecular nitrogen into ammonium nitrogen that can be taken up and utilized by plants (Azarias Guimarães et al. 2012). Therefore, the MDF in our study may affect beneficial bacteria related to pathogen inhibition and nitrogen fixation by interacting with beneficial fungi and bacteria and then influencing microbial networks in the soil.

\section{Effects of the MDF, soil properties, and rhizosphere microbial communities on root nutrient concentration.}

Relative abundance of the MDF varied monthly (Fig. 2C). Changes in abundance of MDF among months affects the abundance of other microorganisms, which in turn affect the structure of the microbial community. The relative abundance of bacteria increases during chitin degradation, which affects the diversity and structure of the soil bacterial community (Jacquiod et al. 2013). In addition, seed mucilage is an input of carbon source into the soil, which affects soil properties. At the same time, mucilage degradation products provide nutrients to plants (Yang et al. 2012a).

The MDF and bacterial communities affect root carbon content (Fig. 4A). Root C content of A. sphaerocephala was lowest in July, which may be due to reduced allocation of plants to underground parts in the growing season. Plants allocate more photosynthetic products to aboveground biomass and less to the belowground biomass in the growing season (Carrara et al. 2018). In May, the high relative abundance of the MDF would increase degradation of seed mucilage, thus making more nutrients available for uptake by roots and thereby increasing root $\mathrm{C}$ content. On the other hand, the degradation products could be utilized by rhizosphere fungal and bacterial communities (Knee et al. 2001).

The abundance of actinobacteria was highest in May. Actinomycetes are bacteria that promote an increase in root nutrients by increasing nutrient availability, regulating plant hormone levels, reducing environmental stress, and inhibiting pathogens (Barka et al. 2016; Hamedi and Mohammadipanah 2015; Palaniyandi et al. 2013). For example, Streptomyces spp. can produce large amounts of hormones (auxin, gibberellins, and cytokinins) that promote plant stem growth (Manulis et al. 1994; Riedlinger et al. 2006; Seipke et al. 2012). Actinomycetes also produce various iron-carrying chelators that bind with $\mathrm{Fe}^{3+}$ from the soil and make it available for plant absorption and utilization (Raymond and Dertz 2004). Rhodococcus spp. (phylum Actinomycetes) can mineralize inorganic phosphorus and release soluble organic phosphorus for plant utilization (Bhatti et al. 2017). Streptomyces spp. increase plant disease resistance by releasing antibiotics and indirectly promoting plant growth (Hamedi and Mohammadipanah 2015; Watve et al. 2001). Therefore, the high abundance of actinomycetes in May suggests that actinomycetes potentially promote the growth of $A$. sphaerocephala and increases roots $\mathrm{C}$ content.

The MDF and rhizosphere fungal communities affect root nitrogen content (Fig. 4A). Overall, the MDF had a positive effect on root nitrogen content (Fig. 4B). In May, the highest relative abundance of the MDF results in the highest root nitrogen content. Inoculation of tobacco (Nicotiana tabacum) with $P$. chrysosporium increases nitrate content in soil by promoting microbial nitrification and increasing nitrate reductase activity (Shang et al. 2017). Therefore, the increase in nitrate content promotes plant nitrogen uptake and increases root nitrogen content in a nitrogen-limited soil such as that in Mu Us Sandland near the Ordos Sandy Grassland Ecological Research Station of the Chinese Academy of Sciences, Inner Mongolia.

In conclusion, we isolated a MDF from the rhizosphere microbes of $A$. sphaerocephala. In the natural habitat, the relative abundance of the MDF and its interactions with closely related microorganisms exhibited seasonal dynamics. The MDF degrades A. sphaerocephala seed mucilage, and its positive interactions with closely related fungi and bacteria might enhance the degradation of $A$. sphaerocephala seed mucilage. In addition, the MDF may regulate root carbon and nitrogen contents by affecting rhizosphere microbial community structure. Therefore, the MDF strengthens plant-soil-microbe interactions that potentially regulate rhizosphere microbial interactions and root nutrients of the cold desert shrub A. sphaerocephala.

\section{MATERIALS AND METHODS}

\section{Collection of the seeds and rhizosphere of A. sphaerocephala.}

In November 2017, freshly matured fruits (achenes) of A. sphaerocephala were collected from a natural population (Supplementary Fig. S1a) growing in moving sand dunes in Ordos, Inner Mongolia, China. Achenes (hereafter seeds) were detached from dry unopened infructescences by manually shaking the plants, and they were then stored dry in paper bags at $4^{\circ} \mathrm{C}$ in the laboratory until used.

Fifteen adult $A$. sphaerocephala plants were randomly selected, and their roots were excavated, in May (beginning of growing season), July, September, and November (end of growing season) 2017, from a natural population growing on moving sand dunes in $\mathrm{Mu}$ Us Sandland. Our sampling was done in different seasons because seed mucilage degradation could last for a long time in the natural habitat. Fine lateral roots ( $\leq 2 \mathrm{~mm}$ diameter) from each plant were sampled and shaken to remove nonrhizosphere soil. Fine roots from the three individual plants were pooled as one root sample. Roots (five replicates $\times$ four seasons) were suspended in sterile distilled water and were shaken for $10 \mathrm{~min}$. The suspension was centrifuged at 3,000 rpm (Sigma centrifuge) for $10 \mathrm{~min}$, and the precipitated rhizosphere soil was divided into two parts, one of which was stored at $5^{\circ} \mathrm{C}$ for screening mucilage-degrading microorganisms and the other at $-80^{\circ} \mathrm{C}$ for DNA extraction. Root $\mathrm{C}$ and $\mathrm{N}$ were determined using an elemental analyzer (Vario EL III, Elementar). 


\section{Isolation of MDF and bacteria from rhizosphere soil.}

Aggregating high potential mucilage-degrading microorganisms. Fifty intact seeds of A. sphaerocephala were placed in a $5-\mathrm{cm}$ diameter Petri dish with $5 \mathrm{ml}$ of distilled water. After the seeds had absorbed water for $10 \mathrm{~min}$, the hydrated seed mucilage (approximately $0.02 \mathrm{~g}$ of dry weight) was stripped from the seed coats using forceps (Supplementary Fig. S1c) (Huang et al. 2008). The main components of seed mucilage were glucose $(29.4 \%)$, mannose $(20.3 \%)$, and arabinose $(19.5 \%)$ (Yang et al. 2012b). The stripped seed mucilage was buried in a glass beaker $(10 \mathrm{ml})$ filled with $5 \mathrm{~g}$ of rhizosphere soil and was incubated in cycles of $20^{\circ} \mathrm{C}$ for $12 \mathrm{~h}$ and $30^{\circ} \mathrm{C}$ for $12 \mathrm{~h}$ (simulating soil temperature fluctuations in the growing season in the natural habitats) in dark to aggregate rhizosphere microorganisms with high potential of degrading mucilage (Khan et al. 2017). The bacterial and fungal strains colonizing the mucilage were identified as having high potential of degrading mucilage. Since seed mucilage had high adhesive ability to soil particles, it formed a large aggregate of particle when contacting sand. Therefore, the large sandy aggregate of particles containing mucilage can be easily separated by sieving the incubation soil with 1-mm mesh. After 2 months of incubation with rhizosphere soil, pectinaceous mucilage covered with sand was sieved out and was washed gently with sterilized water. No antibiotic was added to Luria Bertani (LB) or potato dextreose A (PDA) media. In a preliminary experiment, we had tried to select the mucilage-degrading microorganisms by culturing and screening strains with seed mucilage as the sole carbon source, but microorganisms did not grow. Hence, microorganisms capable of degrading mucilage also need other materials to survive and grow. Therefore, LB and PDA were selected as growth media. Mucilage was then placed on PDA and LB media separately and was incubated at $30^{\circ} \mathrm{C}$ to culture mucilage-degrading microbes. After one week, each colony was transferred to new PDA and LB media for further culture. This transfer was repeated six times to obtain purified mucilagedegrading fungal and bacterial strains.

Screening MDF and bacteria. Seed mucilage reacts with Congo red to form clathrate, and the color fades as the mucilage is degraded. Seed mucilage was stained with a Congo red solution (1\%) for $2 \mathrm{~h}$ and, then, was sterilized at $120^{\circ} \mathrm{C}$ for $2 \mathrm{~h}$. The purified microorganisms were incubated with the stained and sterilized mucilage on PDA and LB solid media. Photographs were taken weekly for 4 weeks to observe the fading Congo red color, which indicates the existence of mucilagedegrading microorganisms.

DNA extraction and sequencing of the MDF. In the above experiment, we found a fungus (its functional role is described above) that was capable of degrading seed mucilage. This MDF was cultured on PDA solid medium for 1 week. MDF DNA was extracted using the fungal DNA extraction kit (Beijing Solarbio Science and Technology Co., Ltd.), following instructions provided with the kit, and was stored at $-20^{\circ} \mathrm{C}$. PCR amplification was carried out using primers ITS1F ( $5^{\prime}$-CTTGGTCA TTTAGAGGAAGTAA-3') and ITS4 (5'-TCCTCCGCTTATT GATATGC-3'), as described by Gardes and Bruns (1993).

Cloning and sequencing the MDF. Cloning was performed using the pEASY-T1 vector kit (Transgen), and the clone was sequenced by a DNA Analyzer (ABI 3730xl, Applied Biosystems Inc.) in both directions.

Phylogenetic analysis. Forward and reverse sequencing were assembled into a consensus sequence, and the vector sequence was removed using VecScreen (available online). Reference sequences were obtained by using BLAST alignment. All sequences were aligned with ClustalW2. Phylogenetic analysis was performed using the MEGA 5.0 program, and the neighbor-joining tree was constructed by performing the
Kimura 2-parameter model with 1,000 bootstrap replicates. The trees were rooted with Candelabrochaete africana.

\section{High-throughput sequencing of rhizosphere microbes.}

To analyze the role of the MDF in the rhizosphere microbial community, high-throughput sequencing of rhizosphere microbial communities was carried out. DNA was extracted from $0.5 \mathrm{~g}$ of rhizosphere soil, using the Power soil DNA isolation kit (MoBio) according to manufacturer instructions, and was stored at $-80^{\circ} \mathrm{C}$. Genomic DNA was amplified with primers 338F (5'-ACTCCTACGGGAGGCAGCAG- $\left.{ }^{\prime}\right)$ and 806R (5'GGACTACHVGGGTWTCTAAT- ${ }^{\prime}$ ) for bacteria and primers ITS1F (5'-CTTGGTCATTTAGAGGAAGTAA- $\left.3^{\prime}\right)$ and ITS2 (5'-GCTGCGTTCTTCATCGATGC-3') for fungi. The PCR products were sequenced on an Illumina MiSeq platform (Illumina), according to manufacturer instructions.

Raw sequences were divided to each sample based on the unique barcodes. The raw paired-end reads were merged by FLASH (Magoč and Salzberg 2011). To obtain high-quality sequences, merged sequences with ambiguous bases or less than $200 \mathrm{bp}$ were filtered by Trimmomatic (Bolger et al. 2014) and chimeric sequences were detected and discarded by UCHIME (Edgar 2010). High-quality sequences were clustered into different OTUs, at the $99 \%$ sequence similarity threshold, by UCLUST (Edgar 2010), to create an OTU table. Taxonomic assignment for each OTU was performed using RDP classifier (Wang et al. 2007) by blasting the sequences from the SILVA database for bacterial communities and the UNITE database for fungi. Sequences that classified into mitochondria and chloroplasts were filtered from the previous OTU table. The final sequences of fungi and bacteria were individually grouped into 869 to 888 and 527 to 1,321 OTUs. The rarefaction curves reached stable plateaus at 20,000 sequences sampled, indicating the sequences depth was sufficient to describe diversity of fungal and bacterial communities (Supplementary Fig. S2). Microbial alpha diversity indexes including OTU richness and the Shannon index were calculated in Mothur (Schloss et al. 2009). One-way analysis of variance (ANOVA) and Tukey honest significant difference statistical tests were performed to compare differences among the four months. Microbial beta diversity was used to determine the dissimilarity of microbial communities across different months. PCoA using the Bray-Curtis distance matrix with the normalized OTU table was performed on the R Phyloseq package to assess the dissimilarity of bacterial and fungal communities between different months. Furthermore, PERMANOVA was conducted to determine whether the dissimilarity of bacterial and fungal communities between different months was significant, using $\mathrm{R}$ vegan package with 999 permutations. The raw sequences were deposited into the National Center for Biotechnology Information Sequence Read Archive database under accession number PRJNA602135. Relative abundance of the MDF in different months was calculated based on the sequencing analysis.

\section{Network construction of rhizosphere microbes.}

To examine fungal-bacterial interactions, networks were constructed based on the relative abundance of OTUs using the Molecular Ecology Network Analysis Pipeline (available online) (Deng et al. 2012). OTUs that were zero in samples were filtered, and missing data were kept blank in the OTU table. Only OTUs detected in three of five samples were used in network construction to ensure reliable correlation networks. Abundance of OTUs was logarithm transformed before similarity matrix construction. A similarity matrix was constructed based on the Pearson correlation coefficient. A proper similarity threshold for pairwise coefficient cut-off between OTUs 
was generated automatically, following the random matrix theory-based method (Zhou et al. 2011). A co-occurrence was identified between a pair of OTUs when the correlation between their abundance exceeded the similarity threshold. Absence of an edge (a zero) meant that two OTUs did not interact directly and were non-co-occurrences. Topological properties of different networks were characterized according to Deng et al. (2012). Major topological properties (i.e., total number of nodes and edges, average path length, degree, and clustering coefficient) of the rhizosphere fungal-bacterial networks were calculated. Finally, networks were visualized by Cytoscape 3.2.0 (Shannon et al. 2003). Subnetworks closely related to the MDF were extracted by the MCODE plug-in in the fungalbacterial networks in different months.

\section{Determination of soil physicochemical properties.}

Soil in the immediate vicinity of the plant was taken to the laboratory, passed through a 2-mm mesh sieve to remove litter and was divided into three subsamples. The first subsample was oven-dried at $105^{\circ} \mathrm{C}$ for $48 \mathrm{~h}$ and was used to determine soil moisture. The second subsample was air-dried and was used to determine soil $\mathrm{pH}$, total $\mathrm{C}$, and total $\mathrm{N}$. The third subsample was stored at $4{ }^{\circ} \mathrm{C}$ and was used for determining soil enzyme activities.

Soil $\mathrm{pH}$ was measured for a suspension of soil and water ( soil/water $=1: 2.5[\mathrm{vol} / \mathrm{wt}])$ by a $\mathrm{pH}$ electrode $(\mathrm{PB}-10$, Sartorius). Total soil $\mathrm{C}$ and $\mathrm{N}$ were determined using an elemental analyzer (Vario EL III). Activities of soil enzymes including phosphatase, $N$-acetyl-glucosaminidase, $\beta$-glucosidase, and cellobiohydrolase were measured using fluorimetric substrates. Detailed procedure for these measurements was previously reported (Hu et al. 2019).

\section{Data analysis.}

Data are expressed as mean \pm standard error. One-way ANOVA was used to compare relative abundance of the MDF in different seasons. The SEM was used to analyze the effects of the MDF, fungal communities, bacterial communities, and soil physicochemical properties on root $\mathrm{C}$ and $\mathrm{N}$. The fungal and bacterial communities were characterized by the first component of PCoA. Correlation analysis between soil physicochemical properties and root $\mathrm{C}$ and $\mathrm{N}$ were carried out, and the significantly relevant variables were incorporated into the SEM. SEM was constructed in Amos software (21.0, SPSS Inc.). Model adequacy was fitted by low $\chi^{2}$ values $(P>0.05)$, high goodness of fit index $(>0.90)$, low Akaike information criteria, and low root mean square errors of approximation $(<0.05)$.

\section{AUTHOR-RECOMMENDED INTERNET RESOURCES}

Molecular Ecology Network Analysis Pipeline: http://ieg4.rccc.ou.edu/mena VecScreen: https://www.ncbi.nlm.nih.gov/tools/vecscreen

\section{LITERATURE CITED}

Aro, N., Pakula, T., and Penttilä, M. 2005. Transcriptional regulation of plant cell wall degradation by filamentous fungi. FEMS Microbiol. Rev. 29:719-739.

Azarias Guimarães, A., Duque Jaramillo, P. M., Simão Abrahão Nóbrega, R., Florentino, L. A., Barroso Silva, K., and de Souza Moreira, F. M. 2012. Genetic and symbiotic diversity of nitrogen-fixing bacteria isolated from agricultural soils in the western Amazon by using cowpea as the trap plant. Appl. Environ. Microbiol. 78:6726-6733.

Baldrian, P., and Valásková, V. 2008. Degradation of cellulose by basidiomycetous fungi. FEMS Microbiol. Rev. 32:501-521.

Banerjee, S., Kirkby, C. A., Schmutter, D., Bissett, A., Kirkegaard, J. A., and Richardson, A. E. 2016. Network analysis reveals functional redundancy and keystone taxa amongst bacterial and fungal communities during organic matter decomposition in an arable soil. Soil Biol. Biochem. 97:188-198

Barka, E. A., Vatsa, P., Sanchez, L., Gaveau-Vaillant, N., Jacquard, C., Meier-Kolthoff, J. P., Klenk, H. P., Clément, C., Ouhdouch, Y., and van Wezel, G. P. 2016. Taxonomy, physiology, and natural products of Actinobacteria. Microbiol. Mol. Biol. Rev. 80:1-43.

Berendsen, R. L., Pieterse, C. M. J., and Bakker, P. A. H. M. 2012. The rhizosphere microbiome and plant health. Trends Plant Sci. 17:478-486.

Bhatti, A. A., Haq, S., and Bhat, R. A. 2017. Actinomycetes benefaction role in soil and plant health. Microb. Pathog. 111:458-467.

Bolger, A. M., Lohse, M., and Usadel, B. 2014. Trimmomatic: A flexible trimmer for Illumina sequence data. Bioinformatics 30:2114-2120.

Carr, A., Diener, C., Baliga, N. S., and Gibbons, S. M. 2019. Use and abuse of correlation analyses in microbial ecology. ISME J. 13:2647-2655.

Carrara, J. E., Walter, C. A., Hawkins, J. S., Peterjohn, W. T., Averill, C., and Brzostek, E. R. 2018. Interactions among plants, bacteria, and fungi reduce extracellular enzyme activities under long-term $\mathrm{N}$ fertilization. Glob. Change Biol. 24:2721-2734.

Cook, R. J., Thomashow, L. S., Weller, D. M., Fujimoto, D., Mazzola, M., Bangera, G., and Kim, D. S. 1995. Molecular mechanisms of defense by rhizobacteria against root disease. Proc. Natl. Acad. Sci. U.S.A. 92: 4197-4201.

Creamer, R. E., Hannula, S. E., Leeuwen, J. P. V., Stone, D., Rutgers, M., Schmelz, R. M., de Ruiter, P. C., Bohse Hendriksen, N., Bolger, T., Bouffaud, M. L., Buee, M., Carvalho, F., Costa, D., Dirilgen, T., Francisco, R., Griffiths, B. S., Griffiths, R., Martin, F., Martins da Silva, P., Mendes, S., Morais, P. V., Pereira, C., Philippot, L., Plassart, P., Redecker, D., Römbke, J., Sousa, J. P., Wouterse, M., and Lemanceau, P 2016. Ecological network analysis reveals the inter-connection between soil biodiversity and ecosystem function as affected by land use across Europe. Appl. Soil Ecol. 97:112-124.

de Boer, W. 2017. Upscaling of fungal-bacterial interactions: From the lab to the field. Curr. Opin. Microbiol. 37:35-41.

de Boer, W., Folman, L. B., Summerbell, R. C., and Boddy, L. 2005. Living in a fungal world: Impact of fungi on soil bacterial niche development FEMS Microbiol. Rev. 29:795-811.

de Graaff, M. A., Classen, A. T., Castro, H. F., and Schadt, C. W. 2010. Labile soil carbon inputs mediate the soil microbial community composition and plant residue decomposition rates. New Phytol. 188:1055-1064.

de Menezes, A. B., Richardson, A. E., and Thrall, P. H. 2017. Linking fungal-bacterial co-occurrences to soil ecosystem function. Curr. Opin. Microbiol. 37:135-141.

Deng, Y., Jiang, Y. H., Yang, Y., He, Z., Luo, F., and Zhou, J. 2012. Molecular ecological network analyses. BMC Bioinformatics 13:113.

Deng, Y., Zhang, P., Qin, Y., Tu, Q., Yang, Y., He, Z., Schadt, C. W., and Zhou, J. 2016. Network succession reveals the importance of competition in response to emulsified vegetable oil amendment for uranium bioremediation. Environ. Microbiol. 18:205-218.

Deveau, A., Bonito, G., Uehling, J., Paoletti, M., Becker, M., Bindschedler, S., Hacquard, S., Hervé, V., Labbé, J., Lastovetsky, O. A., Mieszkin, S., Millet, L. J., Vajna, B., Junier, P., Bonfante, P., Krom, B. P., Olsson, S., van Elsas, J. D., and Wick, L. Y. 2018. Bacterial-fungal interactions: Ecology, mechanisms and challenges. FEMS Microbiol. Rev. 42:335-352.

Edgar, R. C. 2010. Search and clustering orders of magnitude faster than BLAST. Bioinformatics 26:2460-2461.

Faust, K., and Raes, J. 2012. Microbial interactions: From networks to models. Nat. Rev. Microbiol. 10:538-550.

Francoz, E., Ranocha, P., Burlat, V., and Dunand, C. 2015. Arabidopsis seed mucilage secretory cells: Regulation and dynamics. Trends Plant Sci. 20: 515-524.

Gardes, M., and Bruns, T. D. 1993. ITS primers with enhanced specificity for basidiomycetes-Application to the identification of mycorrhizae and rusts. Mol. Ecol. 2:113-118.

Grubert, M. 1974. Studies on the distribution of myxospermy among seeds and fruits of Angiospermae and its ecological importance. Acta Biol. Venez. 8:315-352.

Gutterman, Y., Witztum, A., and Heydecker, W. 1973. Studies of the surfaces of desert plant seeds: II. Ecological adaptations of the seeds of Blepharis persica. Ann. Bot. 37:1051-1055.

Halary, S., Leigh, J. W., Cheaib, B., Lopez, P., and Bapteste, E. 2010. Network analyses structure genetic diversity in independent genetic worlds. Proc. Natl. Acad. Sci. U.S.A. 107:127-132.

Hamedi, J., and Mohammadipanah, F. 2015. Biotechnological application and taxonomical distribution of plant growth promoting actinobacteria J. Ind. Microbiol. Biotechnol. 42:157-171.

Hayat, R., Ali, S., Amara, U., Khalid, R. and Ahmed, I. 2010. Soil beneficial bacteria and their role in plant growth promotion: A review. Ann. Microbiol. 60:579-598. 
Hoppe, B., Kahl, T., Karasch, P., Wubet, T., Bauhus, J., Buscot, F., and Krüger, D. 2014. Network analysis reveals ecological links between N-fixing bacteria and wood-decaying fungi. PLoS One 9:e88141.

Hori, C., Igarashi, K., Katayama, A., and Samejima, M. 2011. Effects of xylan and starch on secretome of the basidiomycete Phanerochaete chrysosporium grown on cellulose. FEMS Microbiol. Lett. 321:1423.

Hu, D., Zhang, S., Baskin, J. M., Baskin, C. C., Wang, Z., Liu, R., Du, J., Yang, X., and Huang, Z. 2019. Seed mucilage interacts with soil microbial community and physiochemical processes to affect seedling emergence on desert sand dunes. Plant Cell Environ. 42:591-605.

Huang, Z., Boubriak, I., Osborne, D. J., Dong, M., and Gutterman, Y. 2008. Possible role of pectin-containing mucilage and dew in repairing embryo DNA of seeds adapted to desert conditions. Ann. Bot. 101:277-283.

Huang, Z., Liu, S., Bradford, K. J., Huxman, T. E., and Venable, D. L. 2016. The contribution of germination functional traits to population dynamics of a desert plant community. Ecology 97:250-261.

Jacquiod, S., Franqueville, L., Cécillon, S., Vogel, T. M., and Simonet, P. 2013. Soil bacterial community shifts after chitin enrichment: An integrative metagenomic approach. PLoS One 8:e79699.

Kameshwar, A. K. S., and Qin, W. 2017. Metadata analysis of Phanerochaete chrysosporium gene expression data identified common cazymes encoding gene expression profiles involved in cellulose and hemicellulose degradation. Int. J. Biol. Sci. 13:85-99.

Khan, S., Nadir, S., Shah, Z. U., Shah, A. A., Karunarathna, S. C., Xu, J., Khan, A., Munir, S., and Hasan, F. 2017. Biodegradation of polyester polyurethane by Aspergillus tubingensis. Environ. Pollut. 225: 469-480.

Knee, E. M., Gong, F. C., Gao, M., Teplitski, M., Jones, A. R., Foxworthy, A., Mort, A. J., and Bauer, W. D. 2001. Root mucilage from pea and its utilization by rhizosphere bacteria as a sole carbon source. Mol. PlantMicrobe Interact 14:775-784.

Liu, S., Bradford, K. J., Huang, Z., and Venable, D. L. 2020. Hydrothermal sensitivities of seed populations underlie fluctuations of dormancy states in an annual plant community. Ecology 101:e02958.

López-Mondéjar, R., Brabcová, V., Stursová, M., Davidová, A., Jansa, J., Cajthaml, T., and Baldrian, P. 2018. Decomposer food web in a deciduous forest shows high share of generalist microorganisms and importance of microbial biomass recycling. ISME J. 12:1768-1778.

Magoč, T., and Salzberg, S. L. 2011. FLASH: Fast length adjustment of short reads to improve genome assemblies. Bioinformatics 27: 2957-2963.

Manulis, S., Shafrir, H., Epstein, E., Lichter, A., and Barash, I. 1994. Biosynthesis of indole-3-acetic acid via the indole-3-acetamide pathway in Streptomyces spp. Microbiol. Read. 140:1045-1050.

Martinez, D., Larrondo, L. F., Putnam, N., Gelpke, M. D., Huang, K., Chapman, J., Helfenbein, K. G., Ramaiya, P., Detter, J. C., Larimer, F., Coutinho, P. M., Henrissat, B., Berka, R., Cullen, D., and Rokhsar, D. 2004. Genome sequence of the lignocellulose degrading fungus Phanerochaete chrysosporium strain RP78. Nat. Biotechnol. 22: 695-700.

McCarthy, A. J. 1987. Lignocellulose-degrading actinomycetes. FEMS Microbiol. Rev. 3:145-163.

Mendes, R., Garbeva, P., and Raaijmakers, J. M. 2013. The rhizosphere microbiome: Significance of plant beneficial, plant pathogenic, and human pathogenic microorganisms. FEMS Microbiol. Rev. 37:634-663.

Mohammadipanah, F., and Wink, J. 2016. Actinobacteria from arid and desert habitats: Diversity and biological activity. Front. Microbiol. 6: 1541.

Oberholster, T., Vikram, S., Cowan, D., and Valverde, A. 2018. Key microbial taxa in the rhizosphere of sorghum and sunflower grown in crop rotation. Sci. Total Environ. 624:530-539.

Palaniyandi, S. A., Yang, S. H., Zhang, L., and Suh, J. W. 2013. Effects of actinobacteria on plant disease suppression and growth promotion. Appl. Microbiol. Biotechnol. 97:9621-9636.

Purahong, W., Wubet, T., Lentendu, G., Schloter, M., Pecyna, M. J., Kapturska, D., Hofrichter, M., Krüger, D., and Buscot, F. 2016. Life in leaf litter: Novel insights into community dynamics of bacteria and fungi during litter decomposition. Mol. Ecol. 25:4059-4074.

Ray, A., Saykhedkar, S., Ayoubi-Canaan, P., Hartson, S. D., Prade, R., and Mort, A. J. 2012. Phanerochaete chrysosporium produces a diverse array of extracellular enzymes when grown on sorghum. Appl. Microbiol. Biotechnol. 93:2075-2089.

Raymond, K. N., and Dertz, E. A. 2004. Biochemical and physical properties of siderophores. Pages 3-17 in: Iron Transport in Bacteria. J.
Crosa, A. Mey, and S. Payne, eds. American Society of Microbiology Press, Washington, D.C.

Riedlinger, J., Schrey, S. D., Tarkka, M. T., Hampp, R., Kapur, M., and Fiedler, H. P. 2006. Auxofuran, a novel metabolite that stimulates the growth of fly agaric, is produced by the mycorrhiza helper bacterium Streptomyces strain AcH 505. Appl. Environ. Microbiol. 72:3550-3557.

Rodríguez, H., and Fraga, R. 1999. Phosphate solubilizing bacteria and their role in plant growth promotion. Biotechnol. Adv. 17:319-339.

Rudnick, M. B., van Veen, J. A., and de Boer, W. 2015. Baiting of rhizosphere bacteria with hyphae of common soil fungi reveals a diverse group of potentially mycophagous secondary consumers. Soil Biol. Biochem. 88:73-82.

Rytioja, J., Hildén, K., Yuzon, J., Hatakka, A., de Vries, R. P., and Mäkelä, M. R. 2014. Plant-polysaccharide-degrading enzymes from basidiomycetes. Microbiol. Mol. Biol. Rev. 78:614-649.

Schloss, P. D., Westcott, S. L., Ryabin, T., Hall, J. R., Hartmann, M., Hollister, E. B., Lesniewski, R. A., Oakley, B. B., Parks, D. H., Robinson, C. J., Sahl, J. W., Stres, B., Thallinger, G. G., Van Horn, D. J., and Weber, C. F. 2009. Introducing mothur: Open-source, platformindependent, community-supported software for describing and comparing microbial communities. Appl. Environ. Microbiol. 75:7537-7541.

Schlüter, A., Heuer, H., Szczepanowski, R., Forney, L. J., Thomas, C. M., Pühler, A., and Top, E. M. 2003. The 64508 bp IncP-1 $\beta$ antibiotic multiresistance plasmid pB10 isolated from a waste-water treatment plant provides evidence for recombination between members of different branches of the IncP-1 $\beta$ group. Microbiol. Read. 149:3139-3153.

Schneider, T., Keiblinger, K. M., Schmid, E., Sterflinger-Gleixner, K. Ellersdorfer, G., Roschitzki, B., Richter, A., Eberl, L., ZechmeisterBoltenstern, S., and Riedel, K. 2012. Who is who in litter decomposition? Metaproteomics reveals major microbial players and their biogeochemical functions. ISME J. 6:1749-1762.

Seipke, R. F., Kaltenpoth, M., and Hutchings, M. I. 2012. Streptomyces as symbionts: An emerging and widespread theme? FEMS Microbiol. Rev. 36:862-876.

Shang, C., Chen, A., Chen, G., Li, H., Guan, S., and He, J. 2017. Microbial biofertilizer decreases nicotine content by improving soil nitrogen supply. Appl. Biochem. Biotechnol. 181:1-14.

Shannon, P., Markiel, A., Ozier, O., Baliga, N. S., Wang, J. T., Ramage, D., Amin, N., Schwikowski, B., and Ideker, T. 2003. Cytoscape: A software environment for integrated models of biomolecular interaction networks. Genome Res. 13:2498-2504.

Shi, S., Nuccio, E. E., Shi, Z. J., He, Z., Zhou, J., and Firestone, M. K. 2016. The interconnected rhizosphere: High network complexity dominates rhizosphere assemblages. Ecol. Lett. 19:926-936.

Vanden Wymelenberg, A., Gaskell, J., Mozuch, M., Kersten, P., Sabat, G., Martinez, D., and Cullen, D. 2009. Transcriptome and secretome analyses of Phanerochaete chrysosporium reveal complex patterns of gene expression. Appl. Environ. Microbiol. 75:4058-4068.

Wang, H. H., Chu, H. L., Dou, Q., Xie, Q. Z., Tang, M., Sung, C. K., and Wang, C. Y. 2018. Phosphorus and nitrogen drive the seasonal dynamics of bacterial communities in pinus forest rhizospheric soil of the Qinling mountains. Front. Microbiol. 9:1930.

Wang, Q., Garrity, G. M., Tiedje, J. M., and Cole, J. R. 2007. Naive Bayesian classifier for rapid assignment of rRNA sequences into the new bacterial taxonomy. Appl. Environ. Microbiol. 73:5261-5267.

Watve, M. G., Tickoo, R., Jog, M. M., and Bhole, B. D. 2001. How many antibiotics are produced by the genus Streptomyces? Arch. Microbiol. 176:386-390.

Yadav, V., and Malanson, G. 2007. Progress in soil organic matter research: Litter decomposition, modelling, monitoring and sequestration. Prog. Phys. Geogr. 31:131-154.

Yang, X., Baskin, C. C., Baskin, J. M., Zhang, W., and Huang, Z. 2012b. Degradation of seed mucilage by soil microflora promotes early seedling growth of a desert sand dune plant. Plant Cell Environ. 35:872-883.

Yang, X., Baskin, J. M., Baskin, C. C., and Huang, Z. 2012a. More than just a coating: Ecological importance, taxonomic occurrence and phylogenetic relationships of seed coat mucilage. Perspect. Plant Ecol. Evol. Syst. 14:434-442.

Yeager, C. M., Gallegos-Graves, V., Dunbar, J., Hesse, C. N., Daligault, H., and Kuske, C. R. 2017. Polysaccharide degradation capability of actinomycetales soil isolates from a semiarid grassland of the Colorado Plateau. Appl. Environ. Microbiol. 83:e03020-16.

Zhou, J., Deng, Y., Luo, F., He, Z., and Yang, Y. 2011. Phylogenetic molecular ecological network of soil microbial communities in response to elevated $\mathrm{CO}_{2}$. MBio 2:e0122-11. 